\title{
DIFERENCIAS TERRITORIALES EN LA MORTALIDAD PREMATURA Y EVITABLE EN ESPAÑA
}

\author{
POR \\ AURORA GARCÍA BALLESTEROS \\ ENRIQUE POZO RIVERA \\ ÁNGELA REDONDO GONZÁLEZ
}

\begin{abstract}
La mortalidad ha seguido en España la tendencia de los países desarrollados: lento y continuado descenso de la tasa de mortalidad estandarizada, unido a un aumento de la esperanza media de vida al nacer. En paralelo a esta evolución la estructura tradicional de la mortalidad se ha transformado de manera que España ha entrado ya de lleno en la cuarta fase de la Transición Epidemiológica, es decir en la edad de las patologías sociales y las enfermedades degenerativas tardías (Schofield y otros, 1991; Omran, 1972; Olskansky y Ault, 1986; Picheral, 1989).

Las principales grandes causas de muerte en la España actual son las enfermedades del aparato circulatorio, los tumores, las enfermedades del aparato respiratorio, las del aparato digestivo y las causas externas, es decir una secuencia análoga a la de la mayoría de los países desarrollados. Como en ellos, el aumento de la esperanza media de vida ha ido unido al incremento del peso de las enfermedades degenerativas y crónicas, siendo precisamente la lucha contra este tipo de enfermedades uno de los elementos básicos en la ganancia de años en la esperanza media de vida.
\end{abstract}

El conocimiento de factores de muy diversa índole: económicos, sociales, psicológicos, culturales, medioambientales, biológicos..., es in-

Departamento Geografía Humana. UCM.

Estudios Geográficos

Tomo LXI, $2000, n \cdot \bullet 241$, octubre-diciembre 
dispensable para entender la mortalidad de la España actual. Ahora bien, si en el pasado los factores económicos tuvieron una gran importancia, las últimas décadas han puesto de manifiesto el papel predominante de determinados comportamientos y estilos de vida. Su incidencia está presente en la mayoría de las grandes causas de muerte a través de factores de riesgo como el tabaquismo, el alto consumo de alcohol, el tipo de alimentación, la calidad ambiental de ciertas zonas o lugares de trabajo, el abuso de ciertas drogas prohibidas, el stress o las conductas arriesgadas al volante. Esa «etiología» social también aparece en nuevas patologías infecciosas vinculadas por ejemplo al Sida o en algunas enfermedades tradicionales de carácter social como la tuberculosis, la gota y el tifus o de forma más anecdótica en patologías víricas e infecciosas procedentes principalmente de los países del Tercer Mundo, como paludismo, cólera..., que resurgen como consecuencia de la mayor interrelación entre los diversas regiones del planeta. Es cierto que muchas de estas patologías infecciosas constituyen más estados de morbilidad que causas de mortalidad, pero debilitan el organismo y le predisponen para una mayor incidencia de otras enfermedades.

Por todo ello, la lucha por ganar años de esperanza media de vida con buena calidad se centra en la transformación de los comportamientos psico-sociales y culturales de la población, de forma que disminuya la sobremortalidad de ciertos grupos de edad, por ejemplo entre 15 y 35 años, o entre determinados colectivos sociales. Sin olvidar por ello las posibles ganancias que puede aportar la lucha contra la mortalidad infantil, donde aún hay grupos de riesgo especialmente por lo que se refiere a las anomalías congénitas y al período perinatal, aunque el peso de la misma supone ya sólo el $0,7 \%$ de la mortalidad total.

\section{Mortalidad y esperanza media de vida en el contexto de la Unión Europea}

En relación con la Unión Europea, España se ha caracterizado en los últimos años por un notable incremento de la esperanza media de vida, acompañado de bajas tasas de mortalidad en ambos géneros.

La esperanza media de vida se ha ido elevando en España hasta llegar a 78,8 años en 1995, por lo que ocupa el cuarto lugar en la Unión Europea después de Francia, Suecia e Italia. 
Esta evolución positiva se corresponde con la relativa a la autovaloración de la salud, ya que si bien en 1987 el $67,9 \%$ de la población percibía el estado de su salud como bueno y muy bueno, en 1997 esta cifra se elevó al 73,1\% (Encuesta Nacional de Salud, 1987 y 1997).

España, al igual que otros países de la Europa mediterránea, ha seguido con retraso la misma tendencia reciente en la mortalidad que el resto de países de la Unión Europea. Tras una fase de suave descenso del número de defunciones y la tasa bruta de mortalidad hasta 1982, se inicia una lenta y continuada recuperación de ambos indicadores en relación con el creciente proceso de envejecimiento demográfico, a la vez que continúa el incremento en la esperanza media de vida (Gil Alonso, F y Cabré, A; 1997). Paralela a esta evolución la estructura tradicional de la mortalidad se ha transformado de manera que España ha entrado ya de lleno en la cuarta fase de la transición epidemiológica, es decir en la edad de las patologías sociales y las enfermedades degenerativas tardías (Schofield y otros, 1991; Omran, 1972; Olshansky y Ault, 1986; Picheral, 1989).

El lento crecimiento del número de defunciones y de la tasa bruta de mortalidad no implica un empeoramiento de las condiciones de vida de los españoles. Muy al contrario el empleo de indicadores que minimizan la incidencia de una estructura por edad cada vez más envejecida, como es el caso de la tasa de mortalidad infantil, la esperanza media de vida y la tasa de mortalidad estandarizada, demuestran la continuación del progreso en la lucha contra la muerte. Así la primera ha caído desde el 11,5 por mil en 1982 al 5,5 por mil de 1995 (INE; Movimiento Natural de la Población) y la segunda ha pasado de los 72,5 y 78,6 años de 1980 a los 73,2 y 81,2 años en 1995 para hombres y mujeres respectivamente (INE; Anuario Estadístico), valores que sitúan a España entre las poblaciones más longevas del planeta. Por su parte la tasa de mortalidad estandarizada, que elimina el efecto de la edad, ha descendido en un casi $11 \%$ en los últimos años. En la comparación de la mortalidad estandarizada entre los países de la Unión Europea, España es el tercer país con menor mortalidad detrás de Suecia y Francia.

Todos los datos expuestos anteriormente nos colocan en cuanto a estos indicadores en una posición superior que la que correspondería por el PNB por habitante, por el que ocupamos el puesto número $13 \mathrm{de}$ la Unión Europea, tan solo por delante de Portugal y Grecia, lo que confirma la gran importancia de los factores socioculturales para explicar 
la mortalidad, una vez se ha alcanzado un cierto umbral de desarrollo económico.

La mayor rapidez de este proceso de mejora en España y en otros países mediterráneos (Portugal, Italia y Grecia), combinado con el estancamiento del mismo en los países de la Europa Oriental, ha modificado el mapa europeo de la mortalidad. A la oposición norte-sur de los años sesenta, que reflejaba el atraso de los países mediterraneos fruto de un tardío inicio de la modernización de la mortalidad (Puyol, R., 1996), le sustituye la oeste-este de los años noventa, con una creciente convergencia interna, además, en el seno de la Unión Europea (Noin, D., 1993; Hall, P. y White, P., 1995; Vallin, J., 1984 y 1991; Gómez Redondo, R., 1997).

Utilizando la lista reducida de causas de muerte, las principales causas de la mortalidad en la España actual son las enfermedades del aparato circulatorio, los tumores, las enfermedades del aparato respiratorio, las del aparato digestivo y las causas externas, es decir una secuencia análoga a la de la mayoría de los países de la Unión Europea. Como análoga es, aunque con retraso y diferentes ritmos, la evolución reciente de estas causas. Así desde principio de los años ochenta descienden las tasas de mortalidad de las enfermedades circulatorias, y en los noventa lo hacen las tasas de las digestivas y las causas externas. Por el contrario siguen creciendo las tasas de los tumores y de las enfermedades respiratorias, y lo hacen también otras tasas de causas que en los años setenta tenían un escaso peso (endocrinas, infecciosas, mentales y del sistema nervioso) en paralelo al crecimiento de patologías sociales en las sociedades modernas postindustriales (Genova i Maleras, R., 1994; Puyol, R.; Pozo, E., y Rodríguez, J., 1999).

Por causas de muerte (Clasificación Internacional de Enfermedades, 9. ${ }^{a}$ Revisión) y en comparación con otros países de la Unión Europea, la primera causa de muerte en España en 1995 son los tumores malignos $(25,2 \%)$. Esta cifra coincide con la de otros países mediterráneos, pero difiere de los restantes de la Unión Europea, en los que la primera causa son las enfermedades del corazón, que en España constituyen la segunda causa de muerte $(23,3 \%)$.

La tercera causa de muerte en España son las enfermedades cerebrovasculares (12,2\%), cuya incidencia porcentual si bien es inferior a la de los países del norte y centro de la UE, presenta la misma tendencia descendente que en los países desarrollados. 
La cuarta causa son las enfermedades pulmonares (4,5\%) y la quinta los accidentes no intencionales, que si bien representan el $3,7 \%$ de la mortalidad, suponen la primera causa de muerte en los menores de 45 años; y aunque la tendencia ascendente de esta causa se ha invertido en los años noventa, España tiene una de las tasas de mortalidad más elevadas de la UE, solo superada por Grecia, Luxemburgo y Dinamarca.

Causas de muerte y años potenciales

de vida perdidos en España

En el estudio de la mortalidad es el análisis de las causas de muerte el que más esclarece las relaciones espacio-sociedad. Sin embargo se suele afirmar que la llegada a la cuarta etapa de la transición epidemiológica va acompañada del descenso de las diferencias socioterritoriales ante la muerte. Analizadas en diversas publicaciones citadas en la bibliografía dichas diferencias, utilizando básicamente indicadores como la tasa ajustada de mortalidad, la esperanza de vida al nacer, la tasa de mortalidad infantil, etc., se ha puesto de relieve que si bien la diferenciación territorial ante la muerte se atenúa, en función de la mejora de los indicadores económicos, culturales y sanitarios, persisten aún desigualdades, máxime si descendemos, como han hecho algunos autores, al análisis de las tasas ajustadas de mortalidad para las principales causas de muerte.

Ante la persistencia de estas desigualdades territoriales nos planteamos en este trabajo una serie de interrogantes: ¿hasta qué punto es reducible la actual tasa de mortalidad?, ¿hasta qué punto se pueden seguir ganado años en la esperanza media de vida? Y por otra parte, ¿cuáles son las causas que dificultan el avance de la esperanza media de vida en las distintas regiones españolas? y, por tanto, ¿cuáles son las causas de la mortalidad prematura y evitable?

Para dar respuesta a alguna de estas interrogantes hemos elegido como indicador el de años potenciales de vida perdidos (APVP), que en la versión estandarizada suministrada por el INE, expresa el número de años de vida perdidos por muertes ocurridas antes de los 70 años, es decir muertes que podríamos considerar prematuras dado que la esperanza media de vida al nacer sobrepasa dicha edad (77 años, 73 para los hombres y 81 para las mujeres). 
Este indicador (INE; Movimiento Natural de la Población, 1995) tiene algunas ventajas en relación con otros más utilizados. Así tanto las tasas brutas de mortalidad, como en menor medida las estandarizadas, están afectadas por la fuerte incidencia de las muertes ocurridas a edades avanzadas, por lo que no permiten evaluar de forma sintética el papel de la mortalidad en las edades jóvenes y adultas, sobre la que los servicios sanitarios y la prevención social deben de incidir y combatir.

El indicador de APVP tiene en cuenta los años que una persona deja de vivir al fallecer a una edad que no es la fijada teóricamente como de defunción en ese colectivo. Este indicador se calcula para el intervalo entre 1 y 70 años, lo que supone por un lado prescindir de la mortalidad degenerativa en edades tardías y por otro de la mortalidad infantil. Por supuesto en ambos grupos hay una mortalidad evitable, pero en el caso de la infantil requiere estudios específicos y detallados por la particularidad de la mayoría de sus causas y además, como se ha señalado, su peso en el conjunto es muy reducido. En el caso de la mortalidad en edades elevadas, cuyo peso es muy alto, dada la tendencia a la uniformidad regional de sus tasas y causas, su inclusión impediría la adecuada valoración de la mortalidad prematura y evitable.

Este indicador es además muy sensible a reflejar tanto los resultados de los avances médicos, incluyendo la calidad de la atención a la población y la mejora de los equipamientos sanitarios, como hechos sociales tales como la valoración de la prevención, la mejora en los hábitos alimentarios, la mayor realización de ejercicio físico, etc.

A nivel estatal, la pérdida de años potenciales de vida ha ido disminuyendo, pero en 1995 aún ha supuesto 1.572 .475 años, y una tasa estandarizada de APVP del 44,98\%, frente a 1.729 .056 años y una tasa del $49,29 \%$ en 1991. Pero con significativos contrastes por género: 1.130 .848 años y una tasa del $64,69 \%$ para los hombres y 441.627 años y una tasa del 25,27\% para las mujeres (INE, Movimiento Natural de la Población 1991 y 1995). Esto supone que, como media, los hombres pierden 15,83 años potenciales de vida y las mujeres 14,63 , lo que confirma una vez más la diferente incidencia de muchas patologías, especialmente las de carácter psicosocial, en ambos géneros.

Ahora bien, no interesa tan solo la cifra general, sino especialmente cuales son las causas qué explican la misma (Cuadros I y II). En todas las grandes causas de muerte salvo en la ${ }^{\circ}{ }^{\circ} \mathrm{XI}$ (complicaciones del embarazo), específica de la mujer, y en la n. ${ }^{\circ}$ XIII (sistema osteomuscular), es 
DIFERENCIAS TERRITORIALES EN LA MORTALIDAD PREMATURA...

CuAdro I

CAUSAS DE MUERTE Y AÑOS POTENCIALES DE VIDA PERDIDOS EN ESPAÑA (1995)

\begin{tabular}{lrrrrrr}
\hline Causas & $\begin{array}{c}\text { Total } \\
\text { años } \\
\text { perdidos }\end{array}$ & $\begin{array}{c}\text { \% todas } \\
\text { las }\end{array}$ & $\begin{array}{c}\text { Total } \\
\text { canos } \\
\text { perdidos } \\
\text { hombres }\end{array}$ & $\begin{array}{c}\text { \% todas } \\
\text { las } \\
\text { causas } \\
\text { hombres }\end{array}$ & $\begin{array}{c}\text { Total } \\
\text { años } \\
\text { perdidos } \\
\text { mujeres }\end{array}$ & $\begin{array}{c}\text { \% } \\
\text { todas } \\
\text { las } \\
\text { mujeres }\end{array}$ \\
\hline Todas & 1.572 .475 & 100,00 & 1.130 .848 & 100,00 & 441.627 & 100,50 \\
I & 24.804 & 1,58 & 16.544 & - & 8.259 & 1,87 \\
II & 466.530 & 29,67 & 292.979 & 25,91 & 173.551 & 39,30 \\
III & 214.272 & 13,63 & 167.244 & 14,79 & 47.028 & 10,65 \\
IV & 5.760 & 0,37 & 3.318 & 0,29 & 2.442 & 0,55 \\
V & 9.302 & 0,59 & 6.700 & 0,59 & 2.602 & 0,59 \\
VI & 36.714 & 2,33 & 21.221 & 1,88 & 15.493 & 3,51 \\
VII & 250.037 & 15,90 & 185.988 & 16,45 & 64.049 & 14,50 \\
VIII & 61.714 & 3,92 & 46.664 & 4,13 & 15.055 & 3,41 \\
IX & 84.225 & 5,36 & 63.792 & 5,64 & 20.433 & 4,63 \\
X & 12.795 & 0,81 & 7.291 & 0,64 & 5.503 & 1,25 \\
XI & 412 & 0,03 & - & - & 412 & 0,09 \\
XII & 833 & 0,05 & 432 & 0,04 & 397 & 0,09 \\
XIII & 3.960 & 0,25 & 1.442 & 0,13 & 2.517 & 0,57 \\
XIV & 18.007 & 1,15 & 10.243 & 0,91 & 7.763 & 1,76 \\
XV & 1.011 & 0,06 & 564 & 0,05 & 447 & 0,10 \\
XVI & 23.979 & 1,52 & 18.190 & 1,61 & 5.789 & 1,31 \\
XVII & 358.120 & 22,77 & 288.233 & 25,49 & 69.887 & 15,82 \\
\hline
\end{tabular}

Fuente: INE, Lista reducida de causas de muerte, según la novena revisión de la Clasificación Internacional de Enfermedades. I: E. infecciosas; II: Tumores; III: E. endocrinas; IV: E. de la sangre; V: T. mentales; VI: E. sist. nervioso; VII: E. A. circulatorio; VIII: E. A. respiratorio; IX: E. A. digestivo; X: E. A. genitourinario; XI: Complicaciones embarazo; XII: E. piel; XIII: E. sist. osteomuscular; XIV: Anomalías congénitas; XV: Afec. originadas en el período perinatal; XVI: E. mal definidas; XVII: causas externas.

mayor el número de APVP en los hombres que en las mujeres. Este hecho no se corresponde con el esquema general de la sobremortalidad en España, donde además de la causa citada hay otras en las que la mortalidad es mayor en los hombres (Pozo, E; 1997). Este es el caso de las enfermedades circulatorias, los trastornos mentales y las enfermedades del aparato osteomuscular. Todas ellas se asocian con claridad a la 
CUADRo II

CAUSAS DE MUERTE Y AÑOS POTENCIALES DE VIDA PERDIDOS EN ESPAÑA. TASAS ESTANDARIZADAS (1995)

\begin{tabular}{lcccccc}
\hline Causas & $\begin{array}{c}\text { Tasa } \\
\text { estanda } \\
\text { rizada }\end{array}$ & $\begin{array}{c}\text { Núm. } \\
\text { medio } \\
\text { años } \\
\text { perdidos }\end{array}$ & $\begin{array}{c}\text { Tasa } \\
\text { estanda- } \\
\text { rizada } \\
\text { hombres }\end{array}$ & $\begin{array}{c}\text { Núm. } \\
\text { medio } \\
\text { años } \\
\text { perdidos } \\
\text { hombres }\end{array}$ & $\begin{array}{c}\text { Tasa } \\
\text { estanda- } \\
\text { rizada } \\
\text { mujeres }\end{array}$ & $\begin{array}{c}\text { Núm. } \\
\text { medio } \\
\text { años } \\
\text { perdidos } \\
\text { mujeres }\end{array}$ \\
\hline Todas & 44,98 & 15,47 & 64,69 & 15,83 & 25,27 & 14,63 \\
I & 0,71 & - & 0,95 & 16,76 & 0,47 & 16,20 \\
II & 13,25 & 11,80 & 16,76 & 11,22 & 9,93 & 12,94 \\
III & 6,13 & 27,96 & 9,57 & 29,46 & 2,69 & 23,67 \\
IV & 0,16 & 16,50 & 0,19 & 16,93 & 0,14 & 15,96 \\
V & 0,27 & 14,03 & 0,38 & 14,63 & 0,15 & 12,70 \\
VI & 1,07 & 18,94 & 1,21 & 19,15 & 0,89 & 18,67 \\
VII & 7,15 & 10,74 & 10,64 & 11,14 & 3,66 & 9,72 \\
VIII & 1,77 & 11,22 & 2,67 & 10,83 & 0,86 & 12,63 \\
IX & 2,41 & 12,79 & 3,65 & 13,19 & 1,17 & 11,66 \\
X & 0,37 & 11,17 & 0,42 & 10,74 & 0,31 & 11,81 \\
XI & 0,01 & 37,50 & - & - & 0,02 & 37,50 \\
XII & 0,02 & 13,83 & 0,02 & 14,91 & 0,02 & 12,82 \\
XIII & 0,11 & 12,86 & 0,08 & 13,00 & 0,14 & 12,78 \\
XIV & 0,52 & 44,03 & 0,59 & 44,34 & 0,44 & 43,62 \\
XV & 0,03 & 48,17 & 0,03 & 56,40 & 0,03 & 40,68 \\
XVI & 0,69 & 22,22 & 1,04 & 22,43 & 0,33 & 21,80 \\
XVII & 10,24 & 30,85 & 16,49 & 31,30 & 4,00 & 29,14 \\
\hline
\end{tabular}

Fuente: INE, Lista reducida de causas de muerte, según la novena revisión de la Clasificación Internacional de Enfermedades. I: E. infecciosas; II: Tumores; III: E. endocrinas; IV: E. de la sangre; V: T. mentales; VI: E. sist. nervioso; VII: E. A. circulatorio; VIII: E. A. respiratorio; IX: E. A. digestivo; X: E. A. genitourinario; XI: Complicaciones embarazo; XII: E. piel; XIII: E. sist. osteomuscular; XIV: Anomalías congénitas; XV: Afec. originadas en el período perinatal; XVI: E. mal definidas; XVII: causas externas.

senilidad, estando sobrerepresentadas en los grupos más elevados de la pirámide de edad donde predominan las mujeres, grupos que han quedado al margen de la elaboración del indicador APVP.

La principal causa de la pérdida de años potenciales de vida son los tumores, casi el 30\% de todas las causas, por lo que la labor de preven- 
ción en este campo debería de ser una de las prioridades de los actuales sistemas de salud estatal y autonómicos. A continuación se sitúan las causas externas que originan la pérdida de 358.120 años, el $22,8 \%$, las enfermedades del aparato circulatorio con 250.037 años perdidos, el 15,9\%, y las enfermedades de las glándulas endocrinas, de la nutrición, del metabolismo y los trastornos de la inmunidad con una pérdida de 214.272 años (el 13,6\%). El resto de las causas tienen valores mucho más bajos destacando en cuarto y quinto lugar las enfermedades del aparato digestivo, entre las que se encuentra la cirrosis, muy relacionada con el consumo de alcohol, y las del aparato respiratorio, en parte viculadas a factores medioambientales, pero también a procesos de debilidad del organismo ligados a enfermedades como el Sida (Cuadro I).

La estructura por género de los APVP muestra un orden de importancia de las causas de muerte semejante al señalado anteriormente, pero con diferencias significativas en la intensidad de los valores. En los hombres hay un mayor equilibrio ya que el n. ${ }^{\circ}$ de APVP por causas externas tiene un peso tan importante como en los tumores. Por otra parte el n. ${ }^{\circ}$ de APVP de las enfermedades endocrinas, en las que se incluye el Sida, alcanza un valor también similar a los años perdidos en las enfermedades del aparato circulatorio. En ambas causas se pone de manifiesto la mayor exposición de los hombres a los factores de riesgo con los que se relacionan estas enfermedades. En las mujeres, por el contrario, el desequilibrio es evidente, pues los tumores son los responsables del 39,3\% de los APVP, situándose a mucha más distancia que en los hombres las causas externas, las enfermedades del aparato circulatorio y las endocrinas (Cuadro I).

Las tasas estandarizadas de APVP a nivel nacional (Cuadro II) ponen de manifiesto que aún se pueden ganar al menos 15,47 años (15,83 en los hombres y 14,63 en las mujeres que parten de una mayor esperanza media de vida) y que esta ganancia en el caso de algunas causas que afectan a grupos reducidos de población puede ser muy significativa. Así en el caso de las anomalías congénitas y las enfermedades originadas en el período perinatal (años perdidos 44,03 y 48,13 respectivamente), cuya mortalidad los avances de la medicina retrasan al menos hasta el primer año de vida. Entre las causas que más afectan a la pérdida de años potenciales de vida en la población femenina joven y adulta están todavía las complicaciones en el embarazo, parto y puerperio, incluyendo el aborto, causa que si bien supone un porcentaje reducido en el conjunto 
total, afecta de forma importante a un grupo social por lo que es necesario actuar para reducir su impacto. Por último para ambos géneros destaquemos las pérdidas por traumatismos, enfermedades endocrinas, del sistema nervioso e infecciosas.

En síntesis, la lucha por ganar años potenciales de vida se tiene que diseñar de forma diferencial según las causas y género, pero también territorialmenete, ya que la incidencia de dichas causas es desigual en las distintas regiones españolas.

Las desigualdades territoriales en la pérdida de años potenciales de vida

El análisis regional descubre importantes diferencias en la mortalidad. En los numerosos trabajos existentes se han estudiado esas diferencias a partir de indicadores como la esperanza media de vida al nacer y las tasas estandarizadas de mortalidad (Cohen, A., 1989 y 1990; Blanco, M., y Farré, M., 1990; Higueras, A., 1990; Faus, M. ${ }^{a}$ C., 1990; Jiménez, R. y otros, 1995; Puyol, R., 1996; Benach, J. y otros, 1996 y 1998; Martínez de Aragón, M. ${ }^{a}$ V, y otros, 1997, etc.). La utilización de la tasa ajustada de APVP descubre así mismo importantes diferencias espaciales, como demuestra la existencia de 15 puntos de distancia entre las regiones en mejor y peor situación, Castilla-La Mancha y Baleares. Ahora bien, el mapa de la mortalidad obtenido con este indicador no coincide exactamente con los que aparecen en los trabajos señalados. Hay un empeoramiento de las regiones más industrializadas y urbanizadas de la mitad norte de España y una mejora de la mala situación de las regiones de la mitad sur; manteniéndose la mayor parte del interior en una buena situación. Evidentemente la propia construcción del indicador APVP contribuye a explicar la no coincidencia en las configuraciones espaciales de la mortalidad. Al considerar solo la mortalidad en las edades de 1 a 70 años se prima la mortalidad de jóvenes y adultos, que alcanza los valores mas altos precisamente en las regiones más industrializadas y urbanizadas de España y los más bajos en el interior y sur del país (González, J., y otros, 1998); por el contrario no se incluye la mortalidad infantil que es aún alta en las regiones del sur y baja en el resto de España (Puyol, R., 1996).

Dentro de ese abanico de valores señalado (15 puntos) se pueden distinguir hasta cuatro tipos de situaciones por sus tasas ajustadas de 
APVP. La más desfavorable corresponde a cuatro regiones que superan el 47\%: Galicia, Asturias, País Vasco y Baleares. Con una situación también mala, pero ya con valores situados algo por encima de la media nacional, aparecen otras cinco regiones: Andalucía, Canarias, Cantabria, Cataluña y Madrid, la única localizada en el interior. El tercer grupo presenta ya una situación que podríamos considerar buena, con una pérdida de años potenciales de vida menor que el valor nacional. Este grupo lo forman Murcia, la Comunidad Valenciana, Aragón y Extremadura. Por último la mejor situación corresponde a Castilla-León, Castilla-La Mancha, La Rioja y Navarra, donde la tasa ajustada de APVP es inferior a 40\% (Fig. 1). El contraste entre las regiones interiores y las litorales, añadiendo Madrid se configura como un rasgo destacado.

Estas cuatro situaciones aparecen de nuevo si realizamos el análisis por género (Fig. 1). La coincidencia es plena o casi plena en quince Comunidades Autónomas. Sólo Andalucía y la Comunidad Valenciana escapan a esta norma. En el caso de Andalucía la situación es especialmente desfavorable en los hombres, mientras que en la Comunidad Valenciana lo es en las mujeres. Estas dos excepciones traducen el

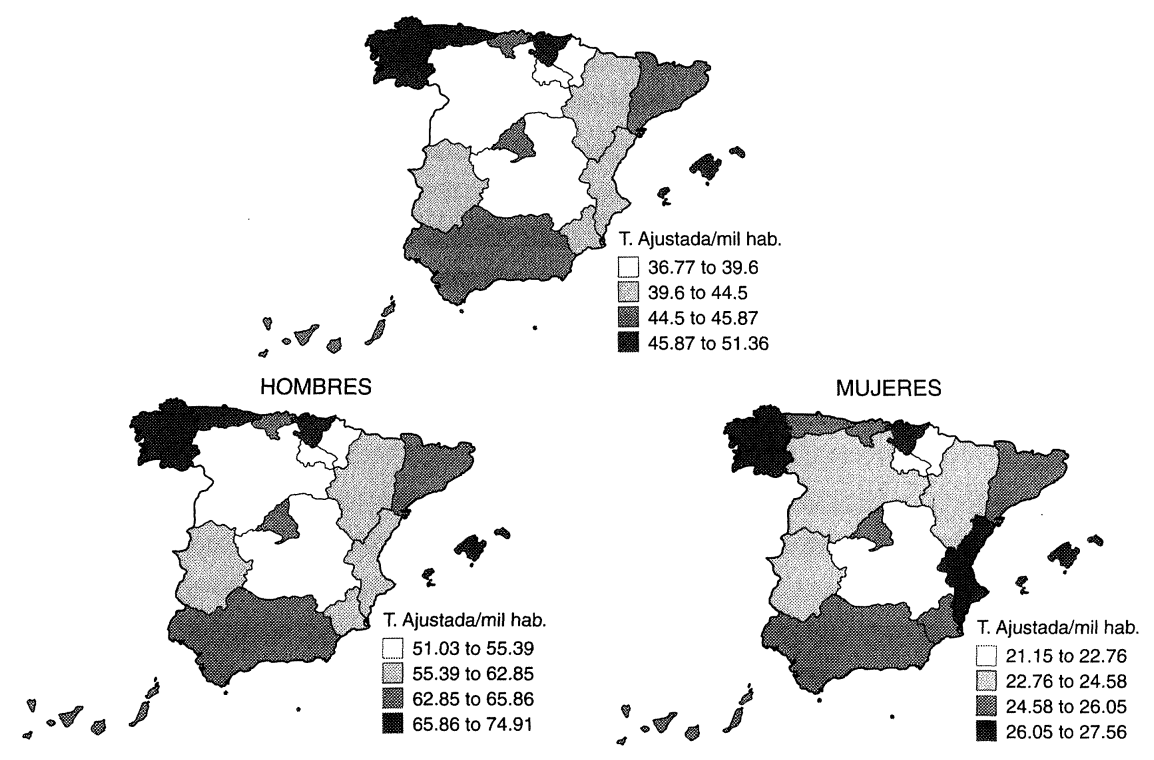

Figura 1.-Años potenciales de vida perdidos. Hombres y mujeres. 
peso diferenciado de las principales causas de muerte por género. En Andalucía, los años potenciales de vida perdidos son superiores al valor nacional en las principales causas de muerte en el caso de los hombres, salvo en las causas externas, mientras que en las mujeres los valores están muy por debajo de la media nacional. En la Comunidad Valenciana sucede lo contrario. Es en las mujeres donde los valores de las tasas de APVP por las principales causas de muerte están por encima del nivel estatal.

En el resto de las Comunidades, las diferencias por género no existen o son menos contrastadas, como en Asturias y Extremadura, donde la menor pérdida de años potenciales de vida en las mujeres hace que su situación, sin variar radicalmente de la de los hombres, sea algo mejor y al contrario en el caso de Castilla-León.

Evidentemente otra cosa es la diferencia por género en la intensidad de los valores, que alcanza cotas apreciables al igual que sucede en otros indicadores de la mortalidad. A nivel estatal hay una distancia de casi 40 puntos en las tasas de APVP de hombres y mujeres. A escala regional, se reproduce este hecho e incluso con valores más elevados. Las mayores diferencias entre ambos géneros se localizan en los focos tradicionales de desarrollo económico, Cataluña, País Vasco y Madrid, seguidos de Galicia, Asturias, Cantabria, Andalucía, Canarias y Baleares. En el polo opuesto se sitúan las regiones de la España interior y la Comunidad Valenciana, donde, como se ha señalado, hay una especial mala situación en las mujeres que convive con una mejor situación relativa de los hombres, lo que se traduce en el acortamiento de la distancia entre los respectivos APVP.

Retomando el análisis conjunto de los dos géneros una primera aproximación explicativa se encuentra en el distinto peso de cada causa de muerte. Para ello hemos elaborado los mapas correspondientes al peso de las seis principales causas de mortalidad, responsables de la perdida del $90 \%$ del total de años potenciales de vida en todas las regiones (Figs. 2 a 7).

Comenzando con las cuatro Comunidades que están en peor situación, o bien en ellas todas las causas consideradas aportan un número de APVP superior a la media nacional, como en Baleares, la comunidad que por ello presenta la situación más desfavorable; o bien, la media es superior en cinco de las seis causas, no existiendo coincidencia en la causa que aporta menos APVP: las enfermedades endocrinas en Galicia, 
las del aparato digestivo en Asturias y las causas externas en el País Vasco.

El grupo de cinco Comunidades con valores altos de APVP, pero en torno a la media nacional, se caracteriza porque esa mala situación aparece en tres o cuatro causas de muerte. No existe coincidencia en ellas pero siempre destaca la presencia de las principales causas de la perdida de años potenciales de vida: tumores, causas externas y enfermedades del aparato circulatorio.

En el caso de las Comunidades donde los APVP son ya menores al valor estatal, también hay dos o tres causas que se sitúan por encima de ese valor. La diferencia con el grupo anterior reside sobre todo en dos hechos: el menor peso de los tumores como causa de muerte y la presencia de solo una de las otras dos grandes causas de muerte.

Por último, las cuatro comunidades en mejor situación destacan por la presencia de bajas tasas en cinco de las seis causas consideradas. Tampoco en este grupo hay coincidencia en la causa restante, es decir en la que presenta valores por encima del nacional, siendo quizás lo más destacable la aparición de las causas externas en este papel en CastillaLeón, Navarra y La Rioja, regiones atravesadas por ejes viarios de gran circulación y alto grado de accidentalidad.

El análisis causa a causa completa el anterior. La principal causa de la perdida de años potenciales de vida en todas las comunidades son los tumores. Los valores más altos se localizan en la España litoral, sobre todo en las regiones del Cantábrico, seguidas por buena parte del litoral mediterráneo, en contraste con los bajos valores de la España interior (Fig. 2).

Con alguna excepción, Cantabria y Navarra, esta misma configuración espacial se repite en las enfermedades del aparato digestivo (Fig. 3), destacando por su mala situación Baleares, Canarias, Andalucía y el País Vasco.

Las causas externas (Fig. 4) constituyen la segunda causa de perdida de años potenciales de vida en la mayoría de las regiones. La configuración espacial es muy distinta que en las anteriores causas, destacando por la alta incidencia Asturias, Galicia y Castilla-León, seguidas por Navarra, La Rioja, Cataluña, Comunidad Valenciana, Murcia y Baleares. Malas infraestructuras en unos casos, alta densidad de tráfico en otros son algunos de los factores explicativos de esta situación.

Las enfermedades del aparato circulatorio (Fig. 5) se sitúan a continuación. La situación más desfavorable se localiza en Extremadura, An- 


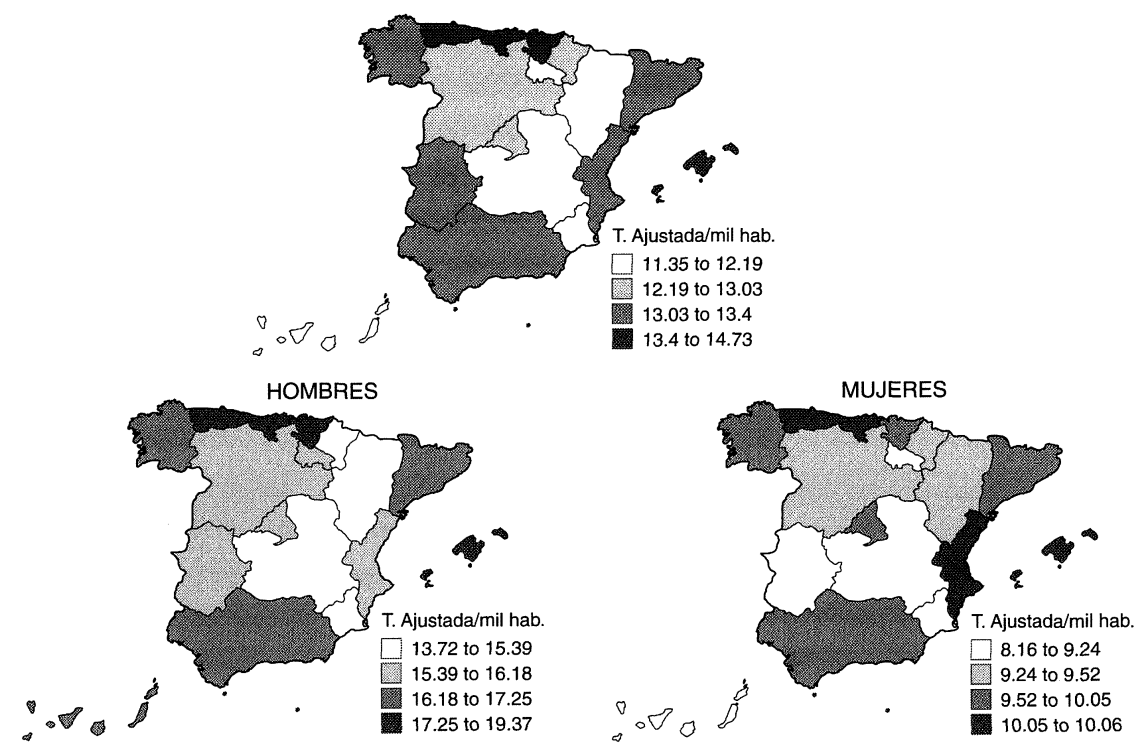

FiguRa 2.-Años potenciales de vida perdidos por tumores.

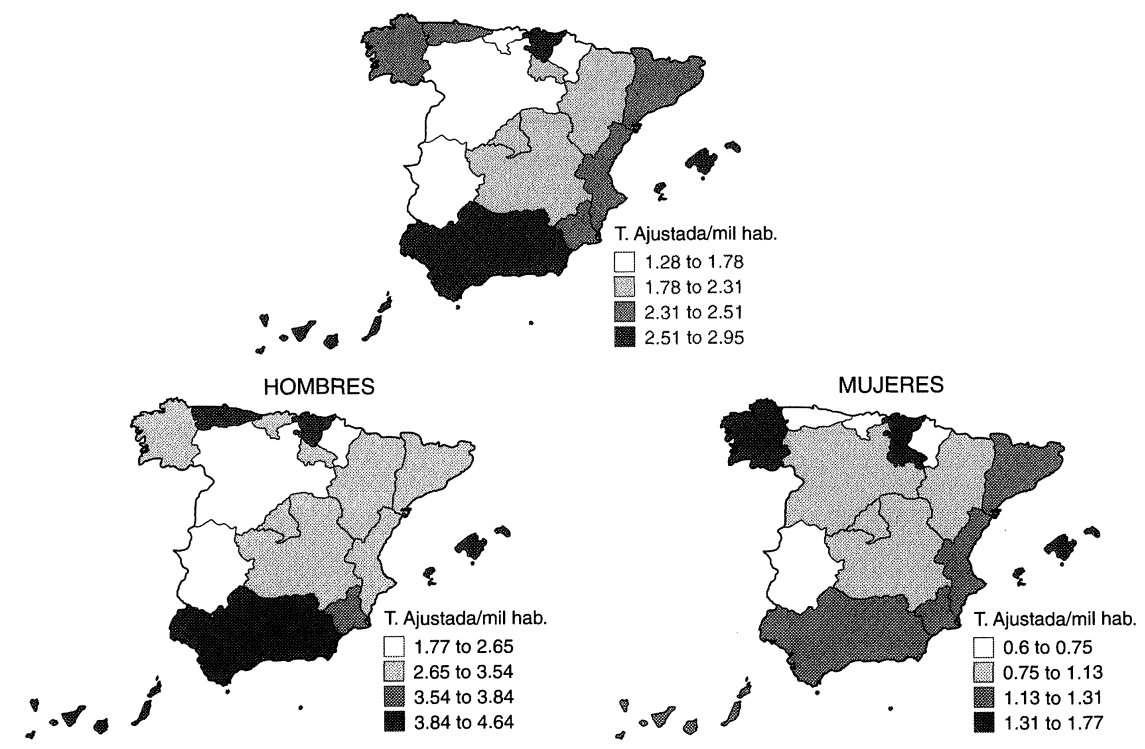

FigURA 3.-Años potenciales de vida perdidos por enfermedades del aparato digestivo.

$$
-640-
$$




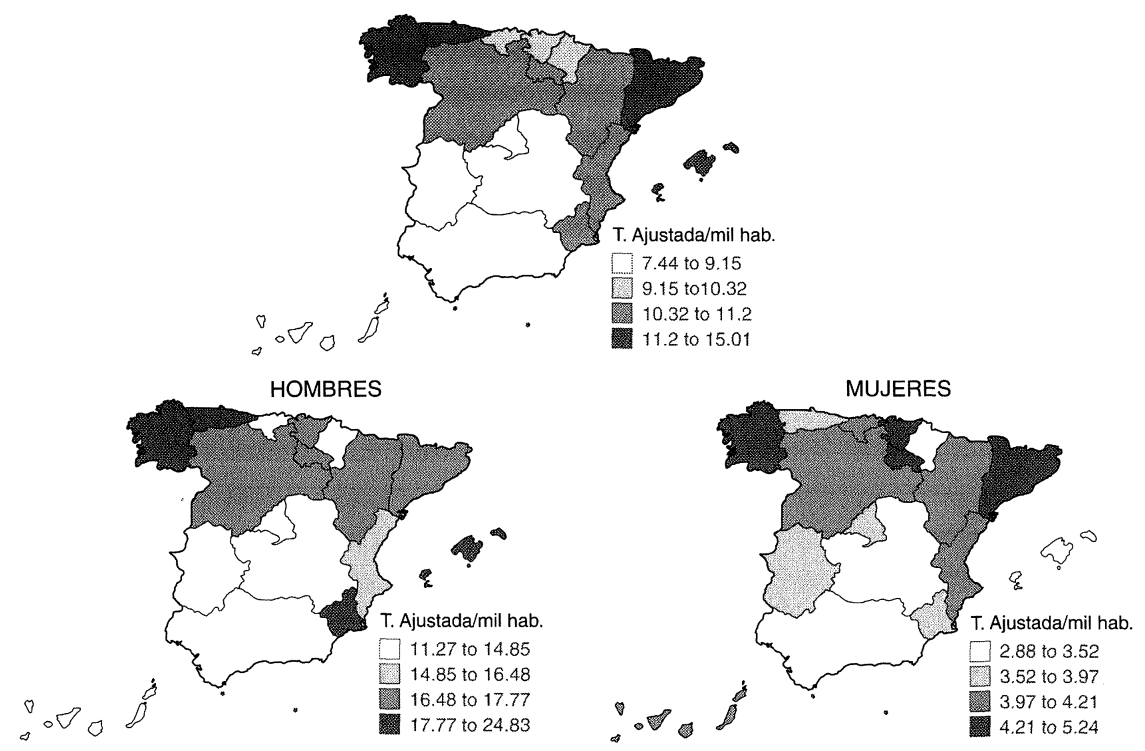

FIGURA 4.-Años potenciales de vida perdidos por causas externas.

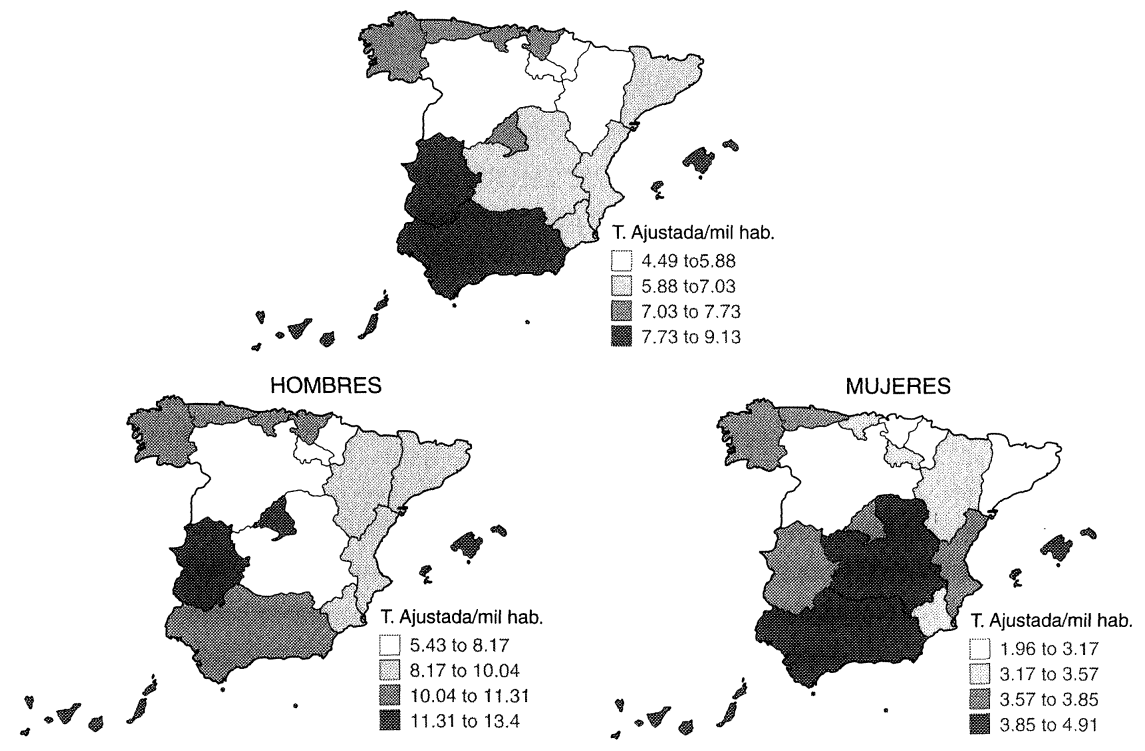

FIGURA 5.-Años potenciales de vida perdidos por enfermedades del aparato circulatorio.

$-641-$ 
dalucía, Canarias y Baleares, seguidas de Galicia, Asturias, Cantabria, País Vasco y Madrid.

La cuarta causa en importancia son las enfermedades endocrinológicas (Fig. 6), cuya configuración regional refleja sobre todo su relación con el nivel de desarrollo económico y altos índices de urbanización. Así los valores más altos están en Cataluña, Madrid y el País Vasco, donde se sitúan incluso en segundo lugar, tras los tumores como causa de perdida de años potenciales de vida. A estas Comunidades les siguen $\mathrm{Ba}$ leares, Comunidad Valenciana, La Rioja y Aragón.

Por último, las enfermedades del aparato respiratorio (Fig. 7) tienen ya un peso muy reducido en la pérdida de años de vida potenciales. La mayor incidencia es en Galicia, Cantabria, Baleares y Madrid, seguidos de Aragón, Cataluña, Extremadura, Andalucía y Canarias.

La explicación de estas desigualdades territoriales nos lleva al análisis de los factores de riesgo con los que están relacionados cada una de las causas. En este sentido se debería profundizar en el análisis de factores como las características de los regímenes alimenticios, el consumo de alcohol y tabaco, la mayor o menor práctica de ejercicio físico, el desarrollo económico, el nivel cultural, los recursos sanitarios, la calidad medioambiental, entre otros.

La diferenciación por género de las distintas causas de pérdida de años potenciales de vida presenta algunos matices de interés, pero de difícil explicación dado que la mayoría de los posibles factores de riesgo de tipo social, cultural, medioambiental, sanitario... no están desagregados de forma que se pueda analizar su incidencia sobre cada género.

En líneas generales, como es lógico, las configuraciones regionales (Figs. 2 a 7) de las principales causas de pérdida de años potenciales de vida en el caso de los hombres se aproximan mucho a las del total de la población que ya hemos analizado. Sin embargo existen algunas excepciones de las que sólo comentaremos las más notables. Así en el caso de los tumores destaca su fuerte peso en Extremadura y en el extremo contrario los bajos valores de la tasa ajustada en Navarra. En las enfermedades del aparato circulatorio la excepción más significativa es Madrid, con una tasa cuatro puntos superior a la general, tal vez vinculada a factores sociales como la mayor tasa de actividad en ciertas profesiones de la población masculina. En las enfermedades del aparato digestivo destaca el valor más alto de Asturias y las tasas más bajas de la mayoría de las regiones del litoral mediterráneo. Sólo un análisis 


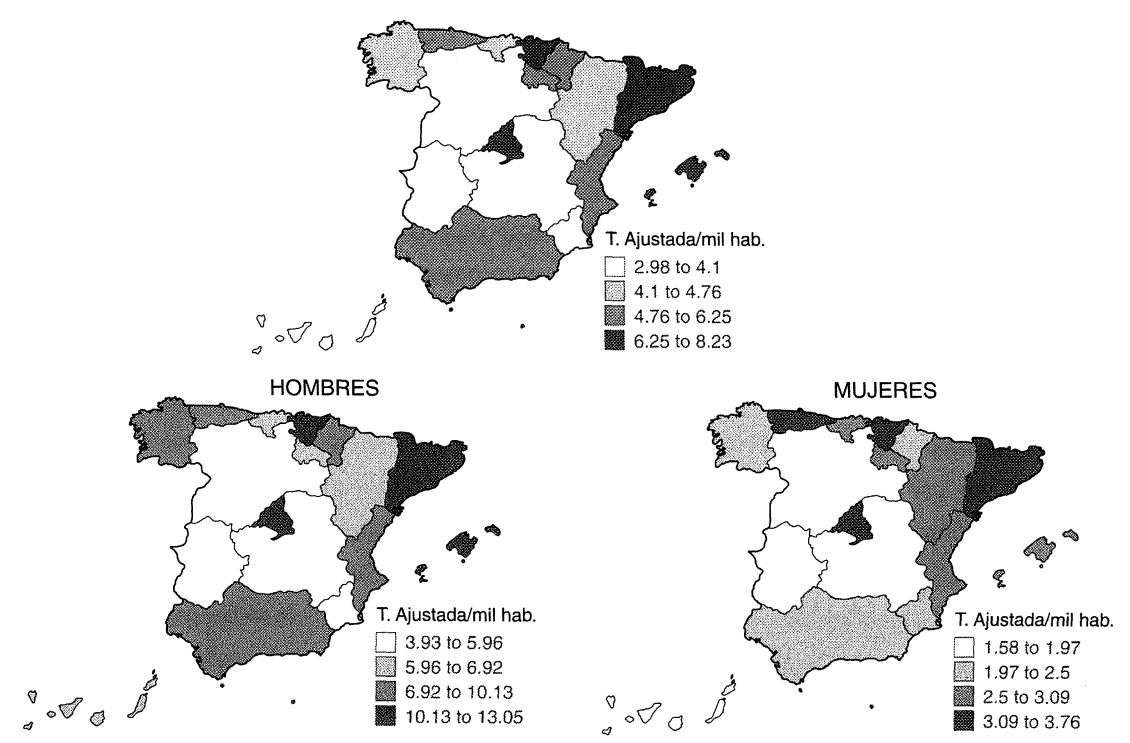

FIGURA 6.-Años potenciales de vida perdidos por causas endocrinológicas.

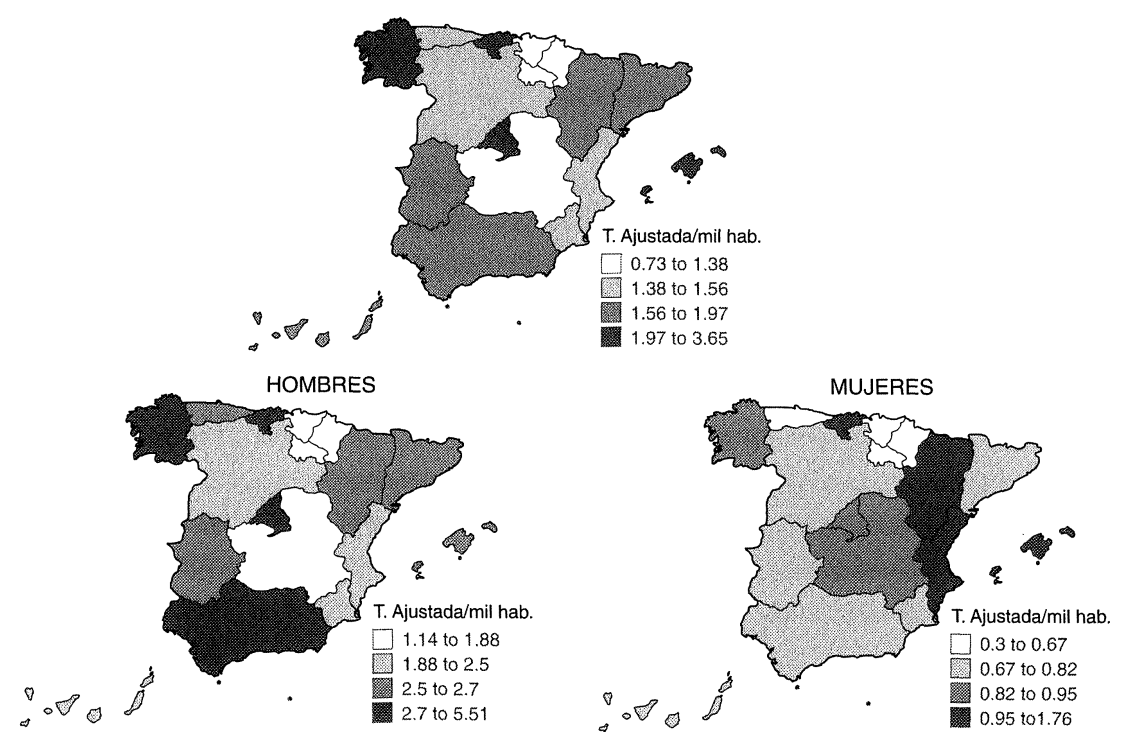

FIGURA 7.-Años potenciales de vida perdidos por enfermedades del aparato respiratorio. 
pormenorizado del consumo de alcohol y tabaco y de la ingesta de lípidos, podría explicar estas situaciones.

En el caso de las mujeres (Figs. 2 a 7), algunas causas están sobrerepresentadas en ciertas regiones. Así en la Comunidad Valenciana, los tumores, sin que tengamos datos sobre la incidencia de las campañas de prevención de los más representativos; en Castilla-La Mancha, las enfermedades del aparato circulatorio; en el País Vasco y La Rioja, las causas externas; en Asturias las enfermedades endocrinas; en Aragón, Comunidad Valenciana y Baleares, las enfermedades respiratorias; en La Rioja y Galicia, las enfermedades del aparato digestivo.

\section{Hacia una geografía de los factores de riesgo}

En relación con la existencia de los principales grupos de enfermedades para los países desarrollados, evidentemente hay una serie de factores causales que inciden directamente sobre ellas. Siguiendo el modelo de Lalonde (1974) cuatro son las variables a considerar cuya interacción determina el nivel de salud de una comunidad: la biología humana (genética y envejecimiento), el medio ambiente (contaminación física, química, biológica, psicosocial y sociocultural), el estilo de vida (conductas de salud) y el sistema de atención sanitaria.

Aunque estos factores son modificables, todos ellos están muy influidos por hechos sociales, ya que la salud y la enfermedad están muy desigualmente distribuidas en la población, debido en gran medida a sus diferencias socioculturales, pues las relaciones entre desigualdades socioterritoriales y salud son más complicadas que la simple relación entre riqueza y salud tal y como se contemplaba tradicionalmente, aunque es cierto que a mayores ventajas sociales corresponden mayores niveles de salud. Como han señalado diversos autores, tanto a escala mundial como en el interior de cada país a escala regional, la magnitud de las desigualdades suele tener una gran importancia. Así en el Reino Unido se ha calculado que si las clases sociales más desfavorecidas tuvieran la misma mortalidad que las de mayor nivel económico, la mortalidad se reduciría en unos 42.000 casos al año (Catalá y de Manuel, 1998). Por tanto políticas sociales y sanitarias pueden reducir de forma considerable los factores de riesgo y la desigualdad ante la enfermedad y la muerte. 
Los estudios epidemiológicos realizados han puesto en evidencia que de las cuatro variables señaladas las dos más importantes, cuyo valor representa las dos terceras partes del total, son el medio ambiente y el estilo de vida, apareciendo los límites entre estas dos determinantes muy imprecisos.

Dever (1977) puso de manifiesto que, cuando se eliminan las muertes ocurridas durante el primer año de vida, en el que los factores biológicos son muy importantes como determinantes de la mortalidad en los países desarrollados, la proporción de mortalidad relacionada con el estilo de vida aumenta acercándose al 50\%, y si el análisis se realiza sobre los años de vida potencialmente perdidos por muertes entre 1 y 65 años, esta proporción supera el valor anterior. Pero en estos estudios no solamente se ha puesto en evidencia el efecto negativo de los comportamientos insanos, medidos en términos de mortalidad prematura, sino también los efectos beneficiosos que para la salud tienen los estilos de vida sanos.

Tres son los hábitos de comportamiento que más se relacionan con la salud y la enfermedad: consumo de tabaco, inactividad física y consumo de alcohol, a los que hay que añadir la obesidad y el número de horas de sueño.

En este trabajo, en línea con los que se realizan actualmente especialmente en el Reino Unido y en Estados Unidos, se pretende hacer un estudio de tipo ecológico, tratando de analizar a escala regional la posible asociación entre indicadores sociales y económicos y las principales causas de muerte que determinan la pérdida de años potenciales de vida. Es cierto que este tipo de trabajos siempre tienen el riesgo de la falacia ecológica, pero también tienen la ventaja de contextualizar territorialmente los factores sociales y ambientales a los que mayoritariamente están expuestas las personas que viven en cada región. Por ello este tipo de estudios son útiles para la planificación de políticas sanitarias y sociales, aunque sería de desear una mayor desagregación de algunos de los indicadores, especialmente por edad y género.

En relación con las causas de la pérdida de años potenciales de vida, los factores de riesgo elegidos han sido los siguientes: con respecto a los hábitos de comportamiento el consumo de tabaco y de alcohol, la ingesta de lípidos y la inactividad física. Por su relación con las características socioeconómicas, de los tres indicadores directos de privación material que señalan Towsend y sus colaboradores (1988) se han elegido 
el nivel de analfabetismo y el índice de hacinamiento, calculado con los valores medianos de la relación entre el número de viviendas con más de una persona por habitación y el total de viviendas principales. La tasa de desempleo se ha sustituido por el índice de pobreza, que de alguna manera sintetiza tanto dicha tasa, como el indicador indirecto de clase social más desfavorecida. De acuerdo con las recomendaciones de la U.E., el INE, en su Encuesta de Presupuestos Familiares, calcula este indicador estableciendo la relación entre el número de hogares cuyo gasto anual medio es inferior al $50 \%$ del gasto medio de los hogares y el número total de hogares. Para matizar los resultados de estos indicadores se ha utilizado también un índice comparativo de nivel de desarrolo, que toma al total de España como base 100 y que por tanto permite ordenar las comunidades autónomas españolas. Por último para medir la relación con el nivel de asistencia sanitaria se ha utilizado el número de camas por cada mil habitantes.

Se ha tenido que prescindir del análisis de la configuración espacial de los factores medioambientales de riesgo, ya que no existe una cobertura suficiente a escala regional de la red de obtención de los datos que miden los parámetro de mayor incidencia en la salud.

En la distribución espacial de la población fumadora (Fig. 8) se observa que, si bien las tasas son elevadas en todo el Estado, hay cuatro comunidades que destacan por sus altas tasas: Cataluña, Baleares, Castilla-La Mancha y Canarias.

En el consumo de bebidas alcohólicas (Fig. 8), al igual que en el caso anterior en casi todas las comunidades se consumen cantidades superiores a la recomendada (60 gramos persona/día), pero son sobre todo Galicia, La Rioja, Cataluña y Murcia, las que sobresalen de forma significativa.

En relación con el consumo de bebidas alcohólicas hay además que tener en cuenta que, según estadísticas recientes (Rubio, 2000), el 40\% de los accidentes de tráfico, con más de dos mil muertos al año, el $46 \%$ de los homicidios y el $25 \%$ de los suicidios y autolesiones, que se producen en España, se deben al consumo de alcohol. Cifras que hay que añadir a las más de 13.000 muertes (unos 224.000 años potenciales de vida perdidos) debidas a enfermedades directamente vinculadas al consumo de bebidas alcohólicas ( $70 \%$ de las cirrosis y otras enfermedades del hígado, unos 5.000 fallecimientos anuales; $42 \%$ de las pancreatitis agudas; $60 \%$ de las pancreatitis crónicas; $75 \%$ de los cánceres de

$$
-646-
$$



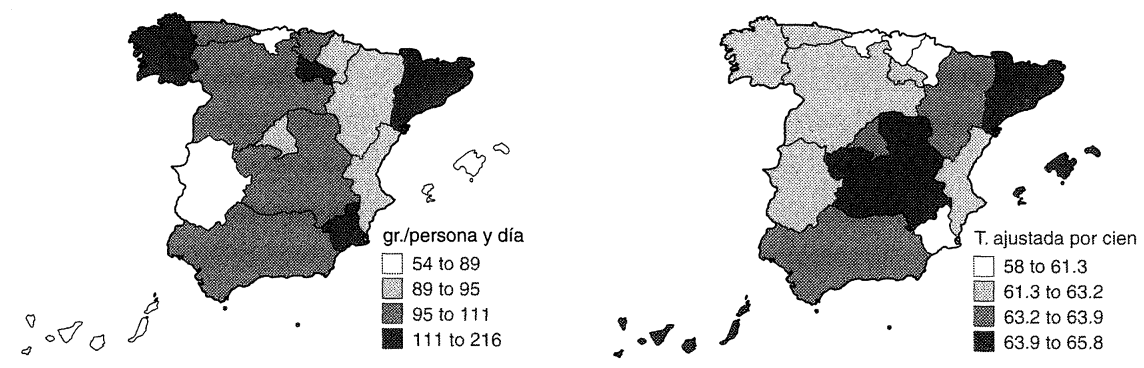

FIgURA 8.-Consumo de bebidas alcohólicas y población fumadora de más de 16 años.

esofago; $50 \%$ de los tumores malignos de la cavidad bucal; $46 \%$ de los cánceres de laringe).

En cuanto a la actividad física, factor muy relacionado con problemas circulatorios y degenerativos, el grado de inactividad física en tiempo libre es un buen indicador (Fig. 9). Los valores más bajos son los de Cantabria, Extremadura y Castilla-La Mancha, por el contrario los más altos se dan en el País Vasco, Asturias, Madrid y Murcia. A este respecto, hay que destacar que en los últimos años, y gracias a la actuación sanitaria en la prevención de enfermedades cardiovasculares, estos valores están disminuyendo, a la par que aumentan los de actividad física con especial incidencia en las mujeres.

El cuarto indicador elegido por su relación con enfermedades circulatorias, endocrinas y degenerativas es el de la ingesta de lípidos
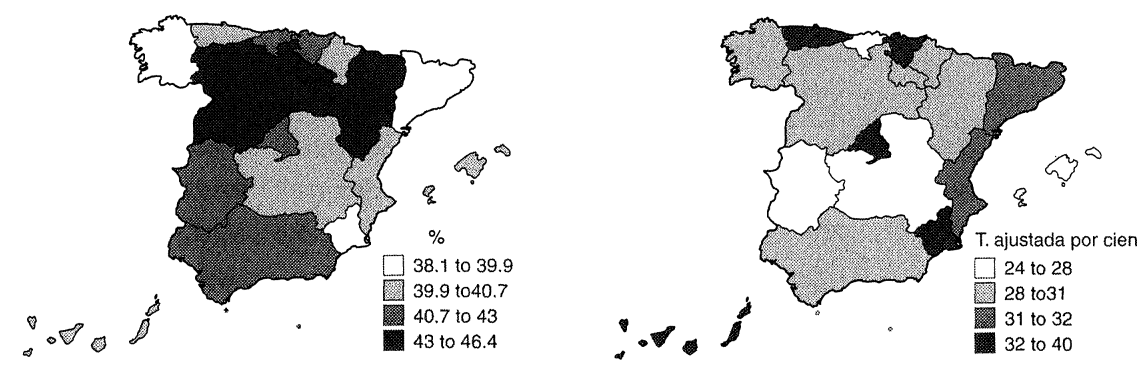

Figura 9.-Ingesta de lípidos por persona y día y población con inactividad fisica en tiempo libre. 
(Fig. 9). También en esta cuestión todas las comunidades superan los valores recomendados (35\% persona/día), destacando por su alto consumo Castilla-León, La Rioja, Navarra y Aragón. Pese a esta situación, en los últimos años se está produciendo una reducción generalizada de los valores de este indicador que explica la tendencia descendente de la mortalidad por enfermedades del aparato circulatorio y degenerativas.

En relación con los indicadores de privación material y de bienestar social, el del grado de analfabetismo (Fig. 10) es bajo en general, pero quedan tres comunidades: Andalucía, Extremadura y Castilla-La Mancha con valores superiores al $5 \%$ de la población.

En relación con la anterior, el índice de hogares pobres (Fig. 10) repite la configuración espacial con la inclusión de Canarias en el grupo de comunidades con valores altos. La misma configuración aparece en el análisis del porcentaje de hacinamiento (Fig. 10) con la incorporación al grupo de altos valores de Galicia y la salida del mismo de Castilla-La Mancha.

Estos indicadores de privación material de alguna manera se sintetizan al calcular el nivel relativo de desarrollo de las Comunidades españolas respecto de la media nacional. En el correspondiente mapa (Fig. 11) destacan los bajos valores de Galicia, Castilla-León, Castilla- La Mancha y Extremadura.

Por último, como indicador de la situación del sistema sanitario que se relaciona normalmente con el $15 \%$ de las causas de muerte, se ha utilizado el número de camas por cada 1.000 habitantes (Fig. 11). En líneas generales la mitad sur de España destaca por su menor dotación, en contraste con la mejor posición de Cataluña, Navarra, Castilla-León y Canarias. Aunque la situación presentada en el mapa ha mejorado en los últimos cinco años, según datos provisionales, todavía ninguna región española alcanza la media de 13 camas por mil habitantes recomendada por la O.M.S., siendo la más cercana a ella Madrid con una media de 10 camas por mil habitantes y persistiendo la desfavorable situación de la mitad sur.

\section{A modo de conclusión}

Del análisis de la situación de las principales causas de perdida de años de vida potenciales y de los principales factores de riesgo se deducen algunas conclusiones: 


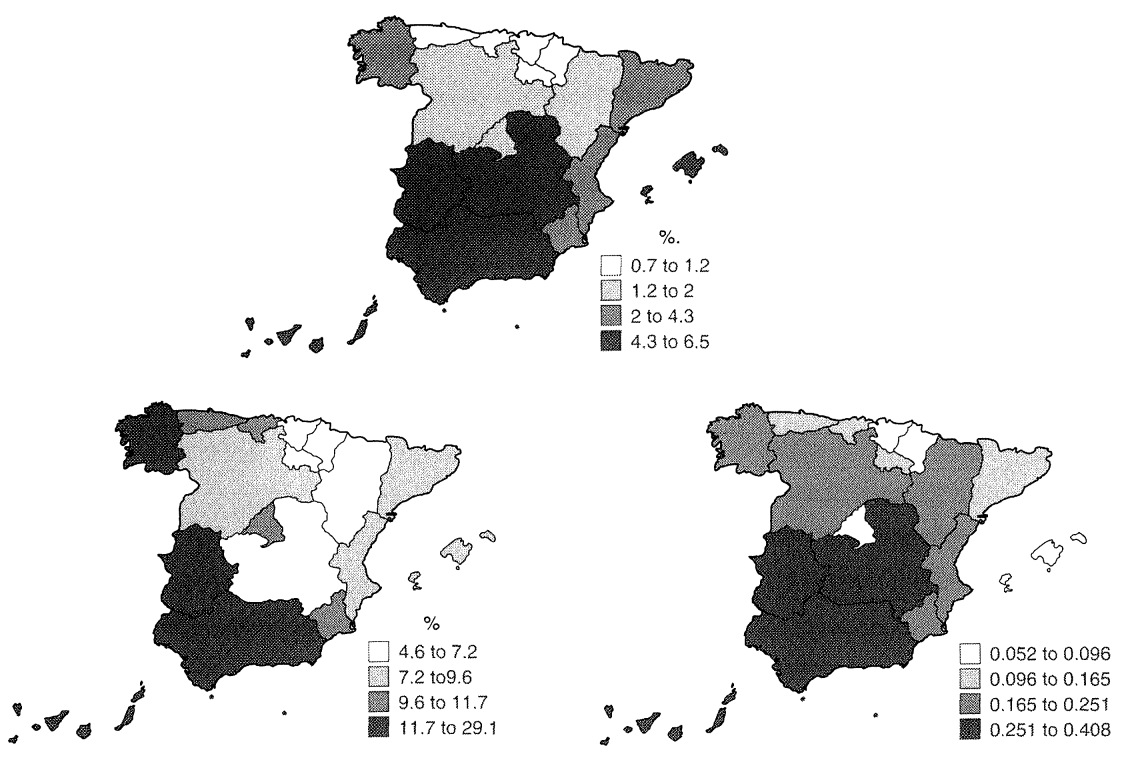

Figura 10.-Población analfabeta. Porcentaje de hacinamiento. Proporción de hogares pobres.
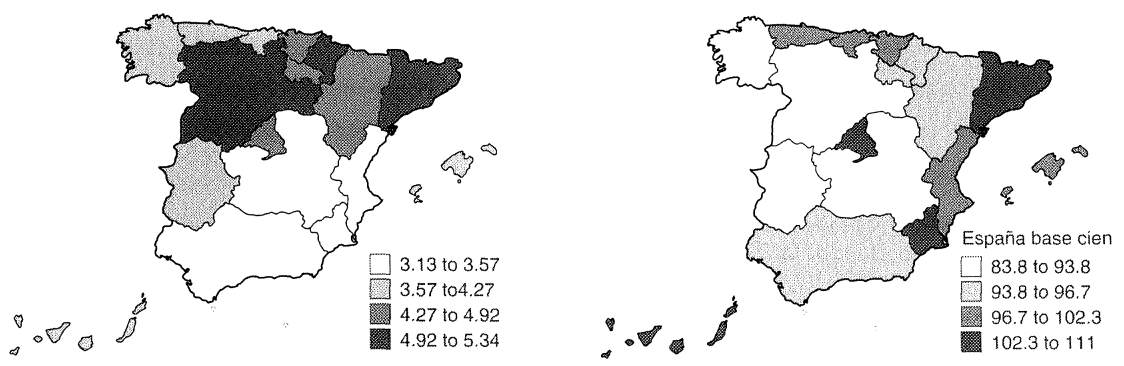

FIGURA 11.-Número de camas por mil habitantes. Nivel de desarrollo.

- La mayoría de las regiones españolas se encuentran, en el contexto de la Unión Europea, en una situación más favorable en los indicadores de mortalidad que la que les correspondería por sus parámetros económicos, en especial por su P.I.B., e incluso por sus indicadores de privación material, como la tasa de desempleo.

$$
-649-
$$


- Por pérdidas de años potenciales de vida la situación es especialmente negativa en Galicia, Asturias, País Vasco y Baleares, por lo que este indicador refleja una situación distinta a la presentada por la tasa ajustada de mortalidad. En efecto, los valores más altos de esta tasa están en Andalucía, Canarias, Comunidad Valenciana y Murcia, aunque es cierto que Baleares y Asturias también superan la media estatal. La reducción en la perdida de años potenciales de vida en las comunidades en que este indicador es más desfavorable contribuirá a la reducción de las desigualdades socioterritoriales en la mortalidad.

- Es aún muy limitado el conocimiento de los mecanismos que pueden explicar la asociación entre los indicadores de privación material y algunas de las causas de muerte. Las configuraciones territoriales de las segundas no se corresponden con claridad a las de las primeras. Ello nos lleva a considerar la necesidad de incrementar los análisis a escala local, en línea con los emprendidos por ejemplo en los últimos años por Benach y colaboradores (1998). Con todo, la mayor posibilidad de exposición a factores de riesgo es siempre un elemento a considerar en la lucha contra la enfermedades y la muerte.

- Las diferencias entre las configuraciones regionales de las principales causas de perdida de años potenciales de vida según género son difíciles de explicar al no disponer de indicadores de factores de riesgo debidamente desagregados. El estudio territorializado de la desigual exposición de hombres y mujeres a las diferentes causas de riesgo, que tiene mucho que ver con su desigual papel en la sociedad, daría datos de interés para una planificación más segmentada de los servicios socio-sanitarios y para una mejor orientación de las campañas de prevención.

- La entrada de todas las regiones españolas en la cuarta etapa de la transición epidemiológica, hace necesario concentrar la lucha en el aumento y mejora de la calidad de la esperanza media de vida y en conseguir el incremento de la buena salud subjetiva que es aún más baja en las mujeres que en los hombres.

- La prevención de la mortalidad accidental, la modificación de factores de riesgo con campañas que contribuyan a cambiar hábitos de comportamiento que se consideran nocivos pero que socialmente están muy arraigados, como el consumo de alcohol o ta- 
baco, o que constituyen pautas culturales, como ciertas dietas alimentarias, o que se imponen como consecuencia de nuevos estilos de vida, como la falta de ejercicio físico, o que se consideran afirmativos de su identidad para ciertos grupos sociales, especialmente de jóvenes, como el consumo de drogas o ciertas formas de conducción, etc, son una vigente necesidad para disminuir la mortalidad en la mayor parte de las regiones españolas, para retrasar la aparición de enfermedades degenerativas y en suma para mejorar su nivel de bienestar.

- Pero también es necesaria una prevención social: mejora en la calidad de las viviendas, planificación de la atención a los grupos sociales que sufren los mayores niveles de privación y por tanto, en principio, más expuestos a factores de riesgo, mejora en la calidad asistencial, especialmente en aquellas regiones más alejadas de las recomendaciones de la O.M.S., mejora en la calidad ambiental del entorno laboral y residencial. En suma, una política social y territorial que preste atención a la situación de los diferentes colectivos sociales y a la mejora de su calidad de vida.

\section{BIBLIOGRAFÍA}

BENACH, J., y otros (1996): «Desigualdades sociales de la mortalidad en España 1990-1992», en NaVARRo, V., y BENACH, J.: Desigualdades sociales en salud en España, Madrid, Ministerio de Sanidad y Consumo.

- (1998): «Desigualdades sociales en mortalidad en áreas pequeñas en España», en CAtalÁ, F., y De Manuel, E.: Ob. cit.

BlANCO, M., y FARRÉ, M. (1990): «Niveaux et causes de mortalité dans les provinces espagnoles», Actes du colloque U.G.I., Lille.

CatalÁ, F., y De MANuEl, E. (1998): Informe S.E.S.P.A.S.: la salud pública y el futuro del estado del bienestar, Granada, Escuela Andaluza de Salud Pública.

GonZÁlez Alonso, J., y otros (1998): «Análisis de la situación de salud», en CATALÁ, F., y DE MANUEL, E. (1998): Informe SESPAS: la salud pública y el futuro del estado del bienestar, Granada, Escuela Andaluza de Salud Pública.

Cohen, A. (1989): «Las disparidades geográficas de la mortalidad en España (19701980)", en A.A.V.V.: Análisis del desarrollo de la población española en el período 1970-1986, Madrid, Síntesis.

- (1990): «La dynamique géographique de la mortalité en Espagne», en Actes du colloque U.G.I., Lille.

Dever, G. E. A. (1977): «Epidemiological model for health policy analisis», Social Indicators Research, pp. 453, 466.

FAus-Pujol, M. ${ }^{a}$ C. (1990): «Morbilité, mortalité en Espagne», en Actes du colloque U.G.I., Lille. 
García Ballesteros,A.; Pozo, E., y Redondo, A.: «La diferenciación espacial de la mortalidad en España», en VI Congreso de Sociología, La Coruña.

GÉNOVA I MALERAS, R. (1994): «Tendencias recientes de la mortalidad en la Comunidad de Madrid», Economía y Sociedad, 10.

GIL AlONSO, F., y CABRÉ, A. (1997): «El crecimiento natural de la población española y sus determinantes», en PuYoL, R. (1997): Dinámica de la Población Española, Madrid, Síntesis.

GómEz REDondo, R. (1995): «Vejez prolongada y juventud menguada. Tendencias en la evolución de la esperanza de vida de la población española, 1970-1990», Revista Española de Investigaciones Sociológicas, 71-72.

- (1997): «La mortalidad de la España actual», Política y Sociedad, 26.

HaLl, P., y White, P. (1995): Europe's Population, Londres, UCL, Press Limited.

HiguERAS, A. (1990): «Mortalité et changement social en Espagne», en Actes du colloque U.G.I., Lille.

INE (1995): Movimiento Natural de la Población, 1995.

INE (1995): Anuario Estadístico. Madrid.

JiMÉNEZ ABOITIZ, R., y otros (1995): «La desigualdad regional ante la muerte», V Congreso Nacional de Sociología, Granada.

LóPEZ ABENTe, G., y otros (1996): Atlas de mortalidad por cáncer y otras causas en España: 1978-1992, Madrid, Instituto de Salud Carlos III.

MaRTínez de ARAgón, M. ${ }^{a}$ V., y otros (1997): Mortalidad en España y Comunidades Autónomas 1989-1992. Situación en la U.E., Madrid, Instituto de Salud Carlos III.

NoIN, D. (1993): «Spatial Inequalities in Mortality», en NoIN, D., y Woods, R. (ed.): The changing population of Europe, Blackwell, London.

Olivera, A. (1996): «Población, salud y bienestar». Treballs de la Societat Catalana de Geografía, 41.

OlshanskY, S. J., y Ault, A. B. (1986): «The fourth stage of the epidemiologic transition: the age of delayed degenerative diseases», The Milbank quarterly, vol. 64 .

OMran, A. R. (1972): «Epidemiologic transition:Theory», en Ross, J. A.(eds.): International Encyclopedia of population, vol I.

Picheral, H. (1989): «Geographie de la transition épidemiologique», Annales de Géographie, 546, pp. 129-151.

Pozo RIvera, E. (1997): «Cambios recientes en la mortalidad y morbilidad en Madrid», Anales de Geografía de la Universidad Complutense, 17.

Puyol Antolín, R. (1996): La Población, Madrid, Síntesis.

Puyol Antolín, R.; Pozo Rivera, E.; Rodríguez Moya, J. (1999): «Evolución y cambios en la población», en GARCíA DELGADO, J. L. (ed): España, Economía: ante el siglo XXI, Espasa-Calpe, Madrid.

Rodríguez, J. A., y LEMKov, L. (1990): «Health and social inequities in Spain», Soc. Sci. Med., 31(3).

RuBio, G. (coord.) (2000): Guía práctica de intervención en el alcholismo, Madrid, Agencia Antidroga de la Comunidad de Madrid y Colegio de Médicos de Madrid.

SCHOFIELD, R., y otros (1991): The decline of mortality in Europe, Oxford, Clarendon Press.

SuRAult, P. (1984): «Les facteurs de differenciationc sociale de la mortalité», Espace, Populations, Sociétés, III.

Towsend, P., y otros (1988): Health and Deprivation: inequality and the North, Londres, Routledge.

VALLin, J. (1984): «Politiques de santé et mortalité dans les pays industrialisés», Espace, Populations,Sociétés, III.

- (1991): «Evolution de la mortalité: hypothése pour les annés futures», Risques, n. ${ }^{\circ} 5$.

- (1995): La población mundial, Madrid, Alianza Ed. 
RESUMEN: El estudio de la mortalidad prematura y evitable a través del indicador de años potenciales de vida perdidos descubre importantes diferencias espaciales en España, que traducen la dispar geografía de los factores de riesgo.En este trabajo se hace una primera aproximación a esta temática con el fin de suministrar pautas sobre las causas que más inciden en la pérdida de anos potenciales de vida en un país que como España ha alcanzado la última fase de la transición epidemiológica.

PALABRAS ClAVE: Geografía de la población, Mortalidad, España, Mortalidad prematura y evitable, Causas mortalidad.

Summary: The study of premature mortality and evitable throw indicator of potential years of live, it lost discover important space differences in Spain, that traduce the uneven geography of the risk factors. In this work it have a first approximation of the thematic, that the end of this is the rules supply of the causes that indicated the lost years of potentials live in a country that like Spain have obtained the last phase of epidemic transition.

KEYS wORDS: Population geography, Mortality, Spain, Premature mortality and evitable, Mortality causes.

RÉSUMÉ: L'étude de la mortalité prématurée et évitable, moyenant l'indicateur d'annés potentielles de vie manquées, met en évidence d'importantes différences territoriales en Espagne, qui font apparaître la dissemblable géographie des facteurs de risque. A cet exposé, on fait une première approximation sur ce sujet afin de dournir des règles sur les causes qui ont une plus grande incidence lors de la perte d'annés potentielles de vie das un pays qui, comme l'espagne, a atteint la dernière phase de la transition épidémilogique.

Most CLEF: Géographie de la Population, Mortalité, Espagne, Mortalité prématuré et évitable, Causes de mortalité. 